\title{
pp32r1 controls the decay of the RNA-binding protein HuR
}

\author{
KENJI IMAMACHI $^{1,2^{*}}$, FUMIHIRO HIGASHINO ${ }^{1 *}$, TETSUYA KITAMURA ${ }^{1 *}$, \\ WATARU KAKUGUCHI ${ }^{3}$, AYA YANAGAWA-MATSUDA ${ }^{1}$, MAKOTO ISHIKAWA ${ }^{2}$, \\ YOSHIMASA KITAGAWA $^{2}$, YASUNORI TOTSUKA ${ }^{3}$ and MASANOBU SHINDOH ${ }^{1}$ \\ Departments of ${ }^{1}$ Oral Pathology and Biology, ${ }^{2}$ Oral Diagnosis and Medicine, ${ }^{3}$ Oral and Maxillofacial Surgery, \\ Hokkaido University Graduate School of Dental Medicine, Sapporo 060-8586, Japan
}

Received September 18, 2013; Accepted November 5, 2013

DOI: 10.3892/or.2013.2956

\begin{abstract}
. pp32 is a tumor suppressor and is one of the associated proteins of the RNA-binding protein HuR. The pp32-HuR complex is exported to the cytoplasm of cells under stress conditions, and HuR is degraded by caspases in the cytoplasm. In the present study, we examined the role of pp32r1, a member of the pp32 family that has oncogenic properties, in the decay of HuR.pp32rl was found to be abundantly expressed in cancer cells, and overexpression of pp32r1 induced colony formation in soft-agar. pp32r1 was expressed in both the nucleus and cytoplasm, whereas pp32 was predominantly localized in the nucleus. Even with lethal stress such as staurosporine (STS), HuR in the cytoplasm was never downregulated, and caspase-3 activity was inhibited when cells expressed pp32r1. pp32r1 bound to HuR without interacting with pp32. In cancer cells, HuR survived in the cytoplasm of cells overexpressing pp32r1, although HuR was not expressed in the cytoplasm of pp32-expressing cells, similar to lethal stress conditions. Taken together, these results indicate that pp32rl binds to HuR to avoid the caspase-mediated decay of HuR in the cytoplasm of cells. We suggest that this function contributes to the oncogenic activity of pp32r1.
\end{abstract}

\section{Introduction}

pp32 (ANP32A) consists of N-terminal leucine-rich repeats (LRR) and a C-terminal acidic domain $(1,2)$. pp32r1 (ANP32C) and pp32r2 (ANP32D) are members of the pp32 family, and genes of this family are located on different chromosomes from that of the pp32 gene (3). Although pp32 has been reported to suppress cellular transformation induced by multiple oncogenes (2), pp32r1 and pp32r2 are the cause

Correspondence to: Dr Fumihiro Higashino, Department of Oral Pathology and Biology, Hokkaido University Graduate School of Dental Medicine, North 13 West 7, Kita-ku, Sapporo 060-8586, Japan E-mail: fhigashi@den.hokudai.ac.jp

${ }^{*}$ Contributed equally

Key words: pp32r1, HuR, decay, pp32, AU-rich element of prostate and breast adenocarcinoma, can transform rodent cells, and produce tumors in nude mice $(3,4)$. pp32 has been shown to enhance apoptotic activity through the stimulation of caspase, which is thought to be required for the tumor suppressive activity of pp32 (5). In cancer cells, phosphorylated retinoblastoma protein forms a complex with pp32 to inhibit pp32-mediated apoptosis, whereas pp32r1 and pp32r2 fail to interact with retinoblastoma protein (6). However, the oncogenic mechanism mediated by pp32r1 and pp32r2 is not fully understood.

pp32 binds to many important proteins such as ataxin-1 (7), Granzyme A (8), the phosphorylated form of $\mathrm{Rb}$ (6) and HuR (9) to modulate their functions. pp32 is involved in the stabilization of the AU-rich element (ARE) containing mRNA through interaction with the RNA-binding protein $\operatorname{HuR}(9,10)$. $\mathrm{HuR}$ is a member of the embryonic lethal abnormal vision (ELAV) family of RNA-binding proteins (11). It can shuttle between the nucleus and the cytoplasm, and the stabilization of ARE-mRNA by HuR is believed to be linked to its localization in the cytoplasm $(12,13)$. HuR is abundant in the cytoplasm in many types of cancer cells, and cytoplasmic HuR expression has been implicated in the malignancy of several types of carcinomas such as colon cancers $(14,15)$.

In a previous study, we identified pp32 as an associated protein of the adenovirus oncogene product E4orf6. It became clear that E4orf6 exported ARE-mRNA from the nucleus to the cytoplasm of cells by interacting with pp32 and HuR protein (16). Moreover, we elucidated that stabilized ARE-mRNA such as $c$-fos and $c$-myc mRNA can contribute to the transformation of cells (17). Cytoplasmic expression of HuR and ARE-mRNA was also abundant in oral cancer cells, which are not caused by virus genes (18), and HuR knockdown downregulated a malignant phenotype such as the invasive activity of oral cancer cells (19). Since ARE-mRNA was found to be exported and stabilized in numerous cancer cells as well (13), the export and stabilization of ARE-mRNA are thought to be general oncogenic mechanisms. To understand the mechanism by which cytoplasmic HuR expression is upregulated in cancer cells, we focused on HuR decay mediated by the pp32 family.

It has been shown that when the HuR-pp32 complex is exported to the cytoplasm by lethal stress such as staurosporine (STS), HuR is cleaved by caspase- 3 and -7 , and the released pp32 activates apoptosomes to induce the apoptosis 
of cells (20). However, no evidence concerning the effects of pp32r1 on HuR regulation has been shown. In this study, we examined the effect of pp32rl on HuR degradation. The expression of pp32r1 in cancer cells was higher than that in normal cells. Unlike pp32, pp32rl was localized to the cytoplasm of cells. pp32r1 inhibited the cleavage of HuR in cells treated with lethal stress. pp32r1 bound to HuR, and HuR survived in the cytoplasm of cancer cells when they expressed pp32rl. These findings suggest that pp32rl binds to HuR to control its decay, and the survival of HuR contributes to the transformation of cells.

\section{Materials and methods}

Cell culture, transfection, stress treatment and cell fractionation. HEK293 (human embryonic kidney cells transformed by the adenovirus E1 gene), SAS (oral cancer), HeLa (cervical cancer), HT1080 (fibrosarcoma), MRC5 (human lung fibroblast), HGF (human gingival fibroblast) and BJ (human foreskin fibroblast) cell lines were obtained from the American Type Culture Collection (ATCC; Manassas, VA, USA) and were cultured at $37^{\circ} \mathrm{C}$ and $5 \% \mathrm{CO}_{2}$ atmosphere in Dulbecco's modified Eagle's medium (DMEM; Sigma) containing $10 \%$ fetal bovine serum (FBS) (HyClone) with antibiotics (Sigma). Cells were transfected with pcDNA3-FLAG-pp32, pCMV2-FLAG-pp32r1 and each empty plasmid (pcDNA3 and pCMV2-FLAG) using Hilymax (Dojindo) according to the manufacturer's protocol. For the lethal stress experiments, cells were treated with $1 \mu \mathrm{M}$ staurosporine (Sigma). In order to separate cells into cytoplasmic and nuclear fractions, cells were resuspended in a fractionating buffer $(10 \mathrm{mM}$ Tris- $\mathrm{HCl}$, pH 7.6, $150 \mathrm{mM} \mathrm{NaCl}, 1.5 \mathrm{mM} \mathrm{MgCl} 2,0.5 \%$ Nonidet P-40, protease inhibitor cocktail), followed by vigorous shaking for $5 \mathrm{~min}$ and centrifugation at $12,000 \mathrm{rpm}$ for $30 \mathrm{sec}$ as previously described (21). The supernatant was used as the cytoplasmic fraction. To estimate the accuracy of cell fractionation, cytoplasmic ( $\gamma$-tubulin) and nuclear (hnRNP A1) proteins were detected by western blotting.

Protein analysis. Western blotting was performed as previously described (22). Antibodies used in this study were specific to HuR, pp32, caspase-3, hnRNP A1 (Santa Cruz), and pp32r1, $\beta$-actin, $\gamma$-tubulin (Sigma). The secondary antibody was horseradish peroxidase-conjugated IgG (Jackson ImmunoResearch Laboratories). Immunoprecipitation was performed as previously described (23). SAS cells were lysed in RIPA buffer [25 mM Tris- $\mathrm{HCl}$ (pH 7.6), $150 \mathrm{mM} \mathrm{NaCl}$, $1 \%$ NP-40, $1 \%$ sodium deoxycholate, $0.1 \%$ SDS] and proteins were immunoprecipitated using anti-pp32 and anti-pp32r1 antibodies.

Immunofluorescence. pp32- and pp32r1-expressing cells plated on coverslips in $35-\mathrm{mm}$ dishes were fixed with $4 \%$ paraformaldehyde, permeabilized with $0.5 \%$ Triton X-100 (Sigma), and incubated with antibodies as follows: mouse monoclonal anti-HuR antibody, goat monoclonal anti-pp32 antibody, rabbit monoclonal anti-pp32r1 antibody. FITCconjugated anti-mouse, rhodamine-conjugated anti-goat or anti-rabbit antibodies were used as secondary antibody. Cells were observed using an IX71 inverted microscope (Olympus).
Soft-agar colony formation assay. pp32-overexpressing or pp32r1-overexpressing cells $\left(3 \times 10^{4}\right)$ were plated per $60-\mathrm{mm}$ culture dish in $3 \mathrm{ml}$ of DMEM containing 10\% FBS and 0.36\% agar on a layer of $5 \mathrm{ml}$ of the same medium containing $0.75 \%$ agar. Three weeks after plating, the colonies were stained with $0.04 \%$ crystal violet- $2 \%$ ethanol in PBS.

\section{Results}

Expression, localization and function of pp32rl in cancer cells. Since it has been demonstrated that pp32r1 can transform cells and that expression of pp32r1 and pp32r2 has been observed in breast and prostate cancer tissues but not in benign tissues $(3,4)$, we determined the expression of the pp32r1 protein in several cancer cell lines: HEK293 (human embryonic kidney cells transformed by the adenovirus E1 gene), SAS (oral cancer), HeLa (cervical cancer), and HT1080 (fibrosarcoma). As shown in Fig. 1A, pp32r1 was expressed in these cancer cells, whereas only slight expression was evident in the normal MRC5 (human lung fibroblast), HGF (human gingival fibroblast) and BJ (human foreskin fibroblast) cells. In contrast, there was only slight difference in pp32 expression between the cancer cells and the normal cells except HGF cells.

To observe the localization of pp32rl, we used an antibody for pp32r1; however, endogenous pp32r1 slightly reacted with it. We then transfected FLAG-tagged pp32 or pp32r1 into SAS oral cancer cells, and the localization of each protein was observed by immunofluorescence using the same antibody. pp32 was mainly expressed in the nucleus, while pp32r1 was localized in the cytoplasm, although it existed both in the nucleus and cytoplasm (Fig. 1B). These results indicate that pp32r1 is abundantly expressed in the cancer cells and is localized in the cytoplasm of the cells.

As pp32r1-induced transformed cells produced tumors in nude mice (3), we examined the anchorage-independent growth activity of pp32r1-expressing cancer cells by the softagar colony formation assay. A FLAG-tagged pp32 or pp32r1 expression construct was introduced into HeLa cells that were then cultured in soft-agar. Numerous colonies were formed by cells with the control plasmid. Cells expressing pp32 were unable to form colonies relative to the control cells, while pp32r1-expressing cells formed large colonies in the soft-agar (Fig. 1C). These results suggest that pp32r1 enhanced the anchorage-independent growth activity of HeLa cells. Taken together these results indicate that pp32 and pp32r1 have totally different properties.

$H u R$ decay in pp32rl-expressing cells upon a lethal stress condition. Upon a lethal stress condition such as staurosporine (STS), HuR and pp32 translocate together to the cytoplasm, and HuR is cleaved into HuR-CP1 and - CP2 by activated caspase- 3 and -7 . The released pp32 activates apoptosomes to induce the apoptosis of cells (20). In order to understand the effect of pp32rl on the cleavage of HuR, SAS cells were transfected with a FLAG-pp32 or FLAGpp32r1 expression vector and cells were treated with STS. The decay and localization of HuR and expression of pp32 and pp32r1 were observed. In the pp32-expressing cells, HuR was localized only in the nucleus, whereas HuR was 
A

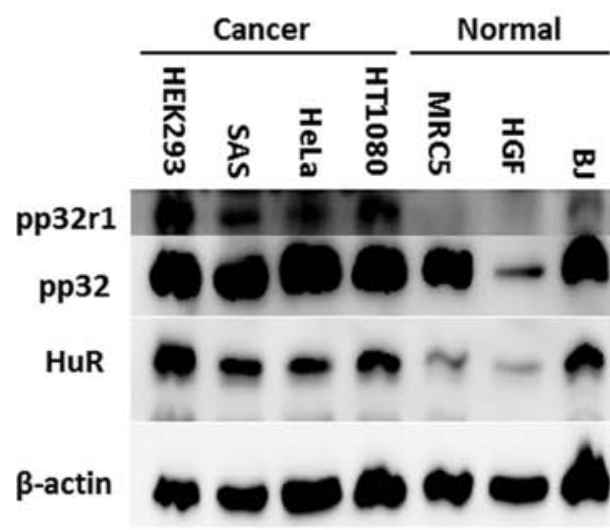

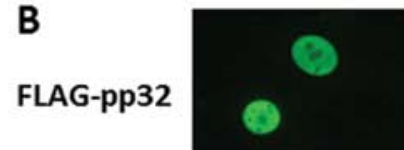

pp32

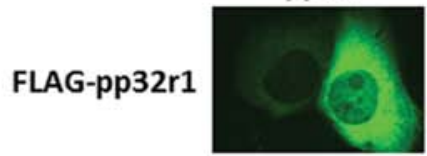

$\operatorname{pp} 32 \mathrm{r} 1$

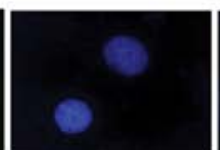

DAPI

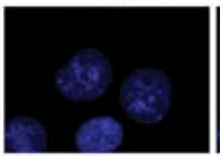

DAPI

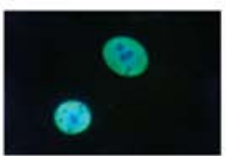

merge

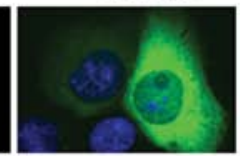

merge

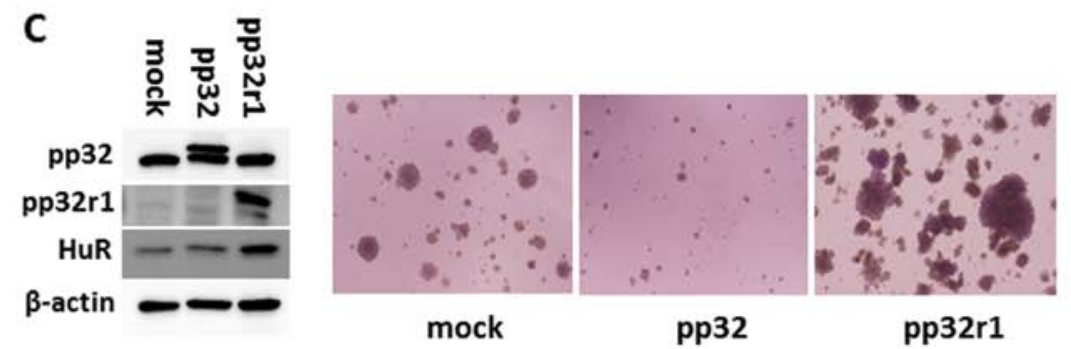

Figure 1. Characterization of pp32 and pp32r1. (A) Expression of pp32,pp32r1 and HuR was examined by western blotting as described (22) using cancer cell lines HEK293, SAS, HeLa, HT1080 and normal cell lines MRC5, HGF and BJ. $\beta$-actin expression is also indicated. (B) Localization of pp32r1 and pp32r1. The expression constructs pcDNA3-FLAG-pp32 and pCMV2-FLAG-pp32r1 were transfected into SAS cells. Cells were incubated with anti-pp32 or anti-pp32r1 antibody followed by FITC-conjugated secondary antibody. DAPI-stained nuclei are shown. (C) The anchorage-independent growth of FLAG-pp32- or FLAGpp32r1-expressing HeLa cells was estimated by a soft-agar colony formation assay. The expression of pp32, pp32r1 and HuR was confirmed by western blotting (left). These cells were plated in soft-agar, and colonies were stained with crystal violet three weeks after plating (right).

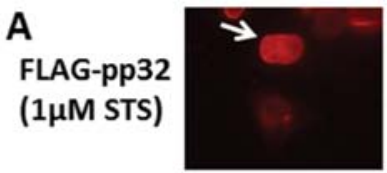

pp32

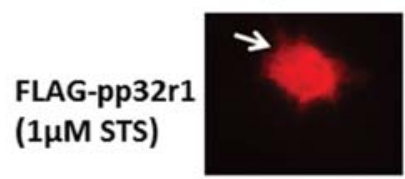

pp32r1

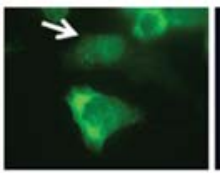

HuR

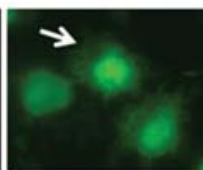

HuR

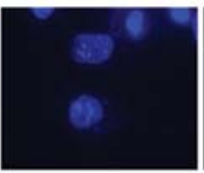

DAPI

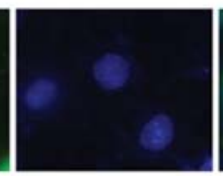

DAPI

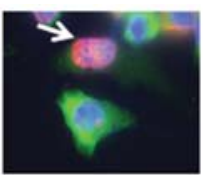

merge

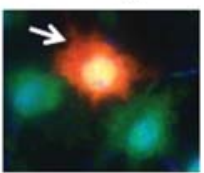

merge
B
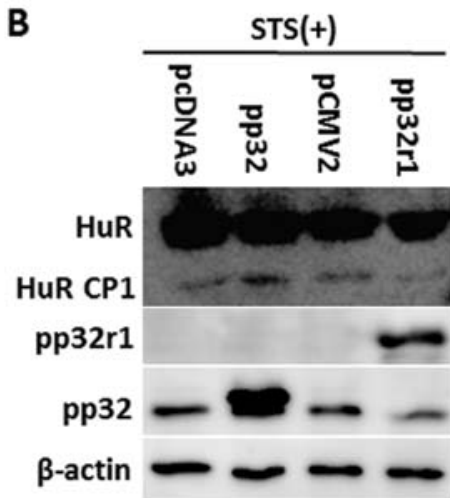

C

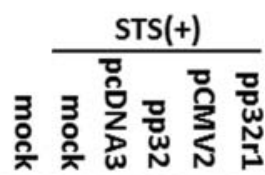

pp32r1

pp32 - - - -

HuR

Caspase-3

(cleaved caspase-3 $\rightarrow$ )

cleaved Caspase-3 $\mathrm{m}=\mathrm{a}=\mathrm{m}=$

$\beta$-actin $---=-$

Figure 2. pp32r1 does not allow the cleavage of HuR in the cytoplasm upon a lethal stress condition. (A) FLAG-pp32 and FLAG-pp32r1 were introduced into SAS cells, and cells were treated with $1 \mu \mathrm{m}$ staurosporine (STS). The localization of HuR, pp32 and pp32r1 was observed as described in Fig. 1B. DAPI-stained nuclei are shown. Arrowheads indicate pp32- and pp32r1-expressing cells. (B) FLAG-pp32, FLAG-pp32r1 and their empty vectors (pcDNA3 and pCMV2FLAG) were transfected into SAS cells, and the expression of HuR-CP1 in each cell line was analyzed by western blotting after treatment with STS. pp32r1, pp32 and $\beta$-actin expression is also indicated. (C) To examine the cleavage of caspase-3, the same transfected cells were treated with STS, and expression of pp32r1, pp32, HuR, caspase-3 and $\beta$-actin was analyzed using western blotting. 

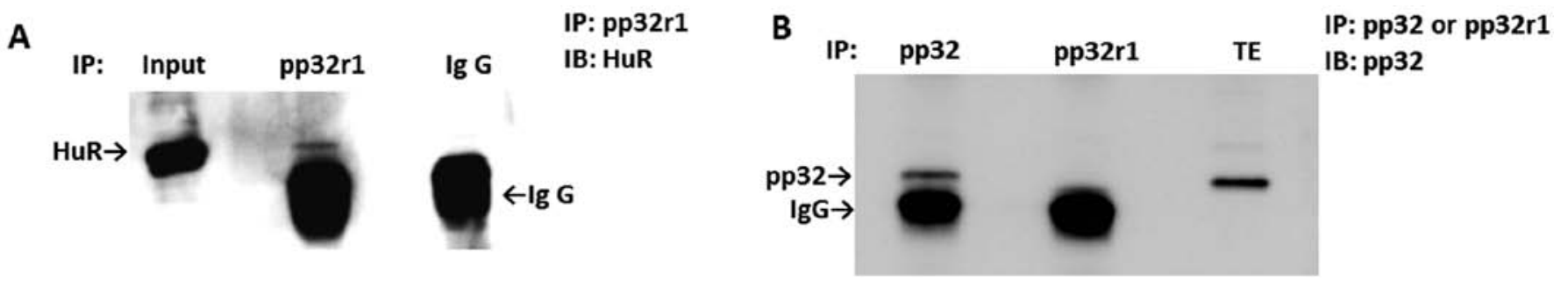

Figure 3. Binding property of pp32r1 to HuR. (A) Interaction between endogenous pp32r1 and HuR. SAS cells were lysed in RIPA buffer, and immunoprecipitation was performed using an antibody for pp32r1. Co-precipitated HuR was analyzed by western blotting. (B) To examine the interaction between pp32 and pp32r1, the SAS cell lysate was immunoprecipitated with an anti-pp32 or anti-pp32r1 antibody, followed by western blotting using the antibody for pp32.

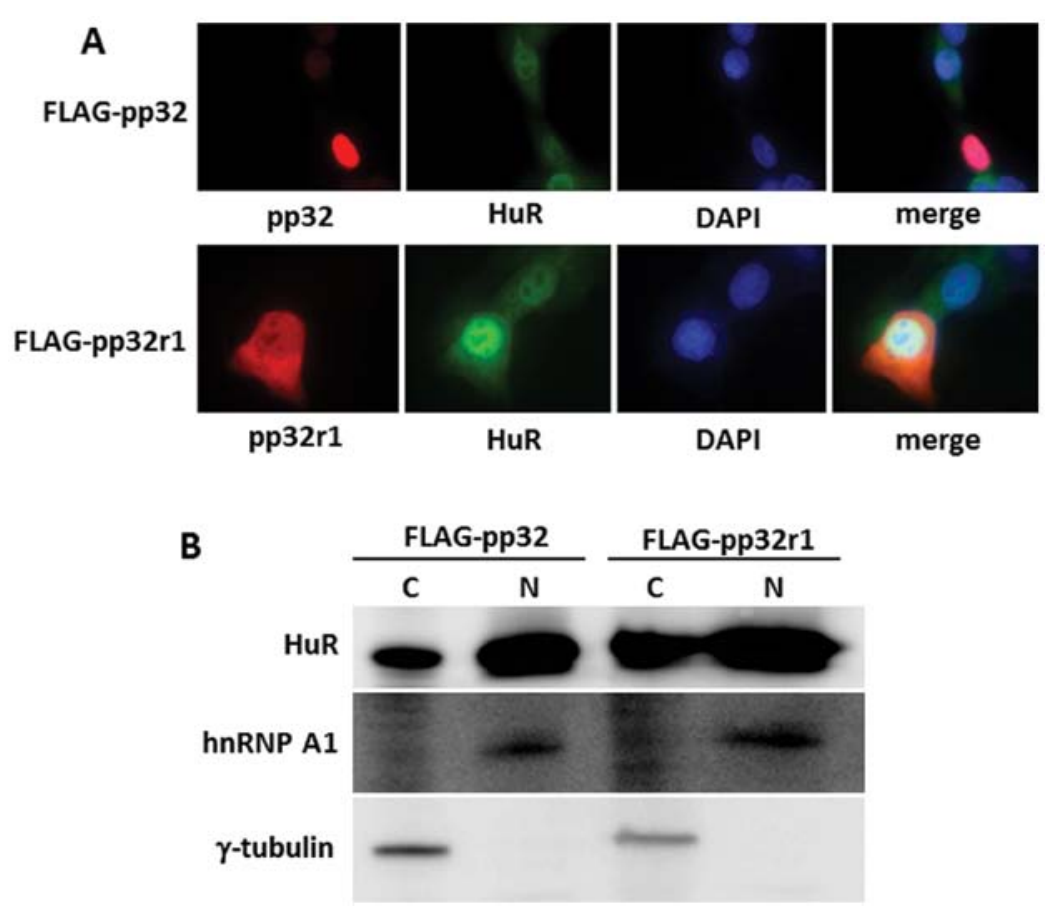

Figure 4. pp32r1 inhibits cleavage of HuR in the cytoplasm of cancer cells. (A) Regulation of HuR cleavage mediated by the pp32 family. FLAG-pp32 and FLAG-pp32r1 expression constructs were introduced into SAS cells, and the amount of HuR in the nucleus and cytoplasm of these cells was estimated by immunofluorescence analysis as indicated in Fig. 2A. (B) The same cells were separated into the nuclear and cytoplasmic fractions, and HuR cleavage in the cytoplasm was estimated by western blotting. To estimate the accuracy of cell fractionation, the cytoplasmic protein $\gamma$-tubulin and nuclear protein hnRNP A1 were detected. $\mathrm{C}$ and $\mathrm{N}$ indicate cytoplasmic and nuclear fractions, respectively.

expressed throughout the cytoplasm of cells expressing pp32r1 (Fig. 2A; arrowheads).

We analyzed the cleaved HuR fragment HuR-CP1 expressed in the same cells using western blot analysis. As shown in Fig. 2B, the amount of HuR-CP1 in pp32r1-expressing cells was decreased compared to that of pp32 expressing cells. These results indicate that HuR cleavage in the cytoplasm of cells under a stress condition was inhibited by the expression of pp32r1.

In a previous study, pp32 was found to activate apoptosome-mediated caspase in the cytoplasm to induce apoptosis, while pp32r1 did not (5). To confirm caspase activity in cells expressing pp32r1, we examined the cleavage of caspase-3 in SAS cells bearing pp32 or pp32r1 plasmids. When a pp32 expression vector was transfected into cells, cleaved caspase-3 was increased by STS stimulation. However, the band declined with pp32r1 expression when compared to cells with the control empty vector even when cells were treated with STS (Fig. 2C). These results indicate that pp32r1 failed to activate caspase in STS-treated cells, which is the reason why HuR protein survived in the cytoplasm of the cancer cells.

pp32rl binds to HuR. Since pp32 is known to be an associated protein of HuR (9), we examined whether or not pp32r1 binds to HuR. To examine the endogenous interactions of these proteins, the lysate of SAS cells was immunoprecipitated with the antibody for pp32r1, followed by western blotting with the anti-HuR antibody. As the HuR band appeared in samples of pp32r1 immunoprecipitation, HuR was co-precipitated with pp32r1 (Fig. 3A). To eliminate the possibility that pp32r1 indirectly binds to HuR mediated by pp32, we examined the binding of pp32r1 with pp32. pp32 was not co-precipitated with pp32r1 (Fig. 3B), indicating that pp32r1 was not able to bind to pp32. These results indicate that pp32r1 interacts with 
HuR similar to the case of pp32 and that binding is not mediated by pp32.

$H u R$ is not degraded in cancer cells expressing pp32rl. As shown in previous studies $(3,4)$ and in Fig. 1A, the expression level of pp32r1 in cancer cells is generally higher than that of normal cells. Furthermore, as pp32rl was found to bind to HuR (Fig. 3A), HuR was hypothesized to bind predominantly to $\mathrm{pp} 32 \mathrm{r} 1$ in cancer cells which inhibits the cleavage of HuR similar to that of the stress condition (Fig. 2). To confirm this hypothesis, we observed HuR cleavage mediated by pp32 and pp32r1 in cancer cells. FLAG-pp32 or FLAG-pp32r1 expression constructs were introduced into SAS cells, and the amount of HuR in the nucleus and cytoplasm of these cells was observed. pp32 expression diminished the expression of HuR in the cytoplasm of SAS cells relative to that in the nontransfected control cells. In contrast, HuR existed throughout the cytoplasm of cells expressing pp32rl similar to the case of the control cells (Fig. 4A). HuR decay in the cytoplasm was then estimated by western blotting. As shown in Fig. 4B, the amount of HuR in the cytoplasm of pp32-expressing SAS cells was obviously less than cytoplasmic HuR expressed in cells with pp32rl. These results suggest that HuR is stabilized in the cytoplasm of cancer cells when they express pp32r1.

\section{Discussion}

In the present study, we compared several properties of pp32 and pp32r1. pp32rl was abundantly expressed in cancer cells whereas its expression was extremely low in normal cells. In contrast, there was only slight difference in pp32 expression. pp32r1 was expressed in the cytoplasm and nucleus of cancer cells, but pp32 existed only in the nucleus. pp32r1 enhanced the anchorage-independent growth properties of cancer cells, whereas pp32 inhibited this ability. pp32 induced the cleavage of HuR in the cytoplasm of cells in a lethal stress condition; however, pp32r1 did not induce it. We confirmed that the binding of pp32r1 with HuR was not mediated by pp32. Additionally, in cancer cells, HuR survived in the cytoplasm of cells expressing pp32r1. Taken together, these findings suggest that when pp32r1 is as abundant in cells as it is in cancer cells, pp32r1 will preferentially associate with HuR and the cytoplasmic expression of HuR may increase through attenuation of caspase-mediated decay.

In a previous study, pp32 was shown to induce apoptosis through the stimulation of Apaf-1-mediated caspase activity. This activity was thought to be required for the tumor suppressive activity of pp32 (5). pp32r1 did not stimulate caspase activity as confirmed in the present study (Fig. 2C). Furthermore, pp32r1 was not able to inhibit the caspase activity of pp32 as caspase activity was not attenuated by adding pp32r1 in an in vitro caspase assay (5). These findings indicate that pp32r1 failed to antagonize the apoptotic activity of pp32 by a competitive manner and that the oncogenic activity of pp32r1 is not implicated in the anti-apoptotic activity. Although pp32r1 and its mutant have been shown to dysregulate the cell cycle (24), the molecular mechanism of the oncogenic activity of pp32r1 remains unclear. In the present study, we demonstrated that pp32rl directly bound to HuR and the cleavage of HuR was inhibited in the cytoplasm of cancer cells where pp32r1 expression was abundantly. We propose that our findings provide one of the explanations for the oncogenic mechanism of pp32r1. Moreover, these data suggest that pp32r1 does not compete with pp32 in cells undergoing apoptosis, but compete with it for HuR stabilization.

We reported that the level of HuR expression was upregulated in the cytoplasm of several types of cancer cells such as oral cancer (18) and viral-mediated transformed cancer (16). Furthermore, the cytoplasmic expression of HuR is evident in a number of human cancers and has been implicated in the malignancy of these carcinomas $(13,18)$. In such cancer cells, several ARE-mRNAs were stabilized in synchrony with cytoplasmic HuR protein levels since HuR binds to ARE and is able to stabilize ARE-mRNA. Although we have no evidence to indicate the relationship between $\mathrm{HuR}$ and pp32r1 in such cancer cells, we suggest that pp32r1 inhibits the decay of HuR in the cytoplasm of these cells and that HuR stabilizes ARE-mRNA to exert its oncogenic activity.

\section{Acknowledgements}

We thank the members of our laboratories for the helpful discussion and support. We also thank Takeshi Kuroshima for his technical advice on immunofluorescence. This work was supported by a Grant-in-Aid for Scientific Research from the Ministry of Education, Science and Culture of Japan.

\section{References}

1. Matsuoka K, Taoka M, Satozawa N, et al: A nuclear factor containing the leucine-rich repeats expressed in murine cerebellar neurons. Proc Natl Acad Sci USA 91: 9670-9674, 1994.

2. Chen TH, Brody JR, Romantsev FE, et al: Structure of pp32, an acidic nuclear protein which inhibits oncogene-induced formation of transformed foci. Mol Biol Cell 12: 2045-2056, 1996.

3. Kadkol SS, Brody JR, Pevsner J, Bai J and Pasternack GR: Modulation of oncogenic potential by alternative gene use in human prostate cancer. Nat Med 5: 275-279, 1999.

4. Kadkol SS, El Naga GA, Brody JR, et al: Expression of pp32 gene family members in breast cancer. Breast Cancer Res Treat 68: 65-73, 2001.

5. Pan W, da Graca LS, Shao Y, Yin Q, Wu H and Jiang X: PHAPI/ pp32 suppresses tumorigenesis by stimulating apoptosis. J Biol Chem 284: 6946-6954, 2009.

6. Adegbola O and Pasternack G: Phosphorylated retinoblastoma protein complexes with pp32 and inhibits pp32-mediated apoptosis. J Biol Chem 280: 15497-15502, 2005.

7. Matilla A, Koshy BT, Cummings CJ, Isobe T, Orr HT and Zoghbi HY: The cerebellar leucine-rich acidic nuclear protein interacts with ataxin-1. Nature 389: 974-978, 1997.

8. Beresford PJ, Kam CM, Powers JC and Lieberman J: Recombinant human granzyme A binds to two putative HLA-associated proteins and cleaves one of them. Proc Natl Acad Sci USA 94: 9285-9290, 1997.

9. Brennan CM, Gallouzi IE and Steitz JA: Protein ligands to HuR modulate its interaction with target mRNA in vivo. J Cell Biol 151: 1-14, 2000.

10. Gallouzi IE, Brennan CM and Steitz JA: Protein ligands mediate the CRM1-dependent export of HuR in response to heat shock. RNA 7: 1348-1361, 2001.

11. Ma WJ, Cheng S, Campbell C, Wright A and Furneaux H: Cloning and characterization of HuR, a ubiquitously expressed Elav-like protein. J Biol Chem 99: 8144-8151, 1996.

12. Hinman MN and Lou H: Diverse molecular functions of $\mathrm{Hu}$ proteins. Cell Mol Life Sci 65: 3168-3181, 2008.

13. Lopez de Silanes I, Lal A and Gorospe M: HuR: post-transcriptional paths to malignancy. RNA Biol 2: 11-13, 2005.

14. López de Silanes I, Fan J, Yang X, et al: Role of the RNA-binding protein HuR in colon carcinogenesis. Oncogene 22: 7146-7154, 2003. 
15. Srikantan S and Gorospe M: HuR function in disease. Front Biosci 17: 189-205, 2012.

16. Higashino F, Aoyagi M, Takahashi A, et al: Adenovirus E4orf6 targets pp32/LANP to control the fate of ARE-containing mRNA by perturbing the CRM1-dependent mechanism. J Cell Biol 170: 15-20, 2005.

17. Kuroshima T, Aoyagi M, Yasuda M, et al: Viral-mediated stabilization of AU-rich element containing mRNA contributes to cell transformation. Oncogene 30: 2912-2920, 2011.

18. Hasegawa H, Kakuguchi W, Kuroshima T, et al: HuR is exported to the cytoplasm in oral cancer cells in a different manner from that of normal cells. Br J Cancer 100: 1943-1948, 2009.

19. Kakuguchi W, Kitamura T, Kuroshima T, et al: HuR knockdown changes the oncogenic potential of oral cancer cells. Mol Cancer Res 8: 520-528, 2010.

20. Mazroui R, Di Marco S, Clair E, et al: Caspase-mediated cleavage of HuR in the cytoplasm contributes to pp32/PHAP-I regulation of apoptosis. J Cell Biol 180: 113-127, 2008.
21. Weigel S and Dobbelstein M: The nuclear export signal within the E4orf6 protein of adenovirus type 5 supports virus replication and cytoplasmic accumulation of viral mRNA. J Virol 74: 764-772, 2000

22. Aoyagi M, Higashino F, Yasuda M, et al: Nuclear export of the adenovirus E4orf6 protein is necessary for its ability to antagonize the apoptotic activity of the $\mathrm{BH} 3$-only proteins. Oncogene 22: 6919-6927, 2003.

23. Higashino F, Pipas JM and Shenk T: Adenovirus E4orf6 oncoprotein modulates the function of the p53-related protein, p73. Proc Natl Acad Sci USA 95: 15683-15687, 1998.

24. Buddaseth S, Göttmann W, Blasczyk R, et al: Dysregulation of cell cycle caused by overexpression of the oncogene pp32r1 (ANP32C) and the Tyr $>$ His mutant pp32r1Y140H. Biochim Biophys Acta 1833: 1212-1221, 2013. 